\title{
NOTE ON PROBABILITY IMPLICATION
}

\section{HANS REICHENBACH}

In a recently published paper ${ }^{1}$ J. C. C. McKinsey has pointed out some difficulties which arise from Axiom I of my theory of probability implication. ${ }^{2}$ This axiom states the unambiguity of the degree $p$ of a given probability implication $\left(O \ni_{p} P\right)$ for the case that the class $O$ is not empty, a condition formulated by $(\overline{\bar{O}})$, but postulates ambiguity of $p$ in case of an empty class $O$, this condition being formulated by $(\bar{O})$. The latter ambiguity is necessary for probability implication because of the relation to Russell's material implication. ${ }^{3}$ From the proof published by McKinsey we can infer that this ambiguity has to be restricted to values of $p$ between 0 and 1, limits included, in correspondence with the same restriction holding for the unambiguous degree $p$ of probability in cases of a non-empty class $O$, formulated by me in $(8, \S 13) .{ }^{4}$ That this general restriction is derivable from Axiom II, 2 is obvious as this axiom contains $O$ and $p$ as free variables and therefore states the restriction for all classes $O$ and all values $p$.

A further objection, which was already indicated in a footnote of McKinsey's paper, has been presented to me in a letter by the referee of this journal, Mr. S. C. Kleene. This objection shows that if the ambiguity of degrees of probability for empty classes $O$ is assumed, it can be proved that this ambiguity cannot be restricted to the limits 0 to 1.

This proof is connected with the theorem of addition (Axiom III) which reads ${ }^{5}$

III. $\left(O \ni_{p} P\right) .\left(O \ni_{q} Q\right) \cdot(O \cdot P \supset \bar{Q}) \supset(\exists r)\left(O \ni_{r} P \vee Q\right) .(r=p+q)$.

The condition $r \leqq 1$ implies that $p+q \leqq 1$. If we demand $r \leqq 1$ only for non-empty classes $O$, the mentioned restriction for $p$ and $q$, which

1 This Bulletin, vol. 45 (1939), pp. 799-800.

2 Published in Wahrscheinlichkeitslehre, Leiden, 1935, $\$ \$ 12-14$. My further quotations refer to this book.

${ }^{3}$ Page 66.

${ }^{4}$ To avoid misunderstandings let me add here the remark that this relation is meant only for the case that the probability $W(O, P)$ exists, and would be written in the implicational writing

$$
\left[(\exists x)\left(O \ni_{x} P\right)\right] \supset\left[(\exists y)\left(O \ni_{y} P\right) .(0 \leqq y \leqq 1)\right] .
$$

If $O$ is not empty and therefore the probability has only one value, this means that this value is restricted to the limits 0 to 1 , limits included.

${ }^{5}$ I write here the existential operator on the right-hand side because the abbreviation introduced on page 62 , according to which the existential operator is omitted in the corresponding formula of my book, may be misleading. 
is stronger than $(8, \S 13)$, need not be stated in the implicans of the axiom because we then rather infer from III that the two conditions

$$
p+q>1, \quad(O . P \supset \bar{Q})
$$

are incompatible. Following the principle that numerical values leading to contradictions are to be excluded, we can thus deduce all numerical restrictions for the values of probabilities; in $(15, \S 19)$ I have developed a formula which states these restrictions for all possible cases. The incompatibility of the two formulae (1) for non-empty classes $O$ is included in $(15, \S 19)$ because this relation shows that if $p+q>1$ we have $W(O . P, Q)>0$, which in consideration of $(9, \S 13)$ contradicts $(O . P \supset \bar{Q})$ in case $O$ is not empty. However in applying the explained principle we have to be sure that the excluded numerical values cannot be introduced by means of other ways of deduction. It turns out that this is the case for an empty class $O$. In this case, that is for $(\bar{O})$, we can for instance assume in III the numerical values $p=1$ and $q=1$ whereas the condition $(O . P \supset \bar{Q})$ is also satisfied; the latter follows from the properties of material implication according to which a false proposition implies every proposition. It follows that in this case $r$ can be greater than 1 .

These difficulties are eliminated if we introduce in Axiom II,2 the condition that $O$ is not empty, and write this axiom:

$$
\text { II,2. } \overline{(\bar{O})} \text {. }\left(O \ni_{p} P\right) \supset(p \geqq 0) \text {. }
$$

With the qualification $\overline{(\bar{O})}$ in the implicans we renounce the unnecessary extension of numerical restrictions to probability values referring to empty classes. It is without danger to assume that in case $O$ is empty the probability $p$ of $\left(O \ni_{p} P\right)$ can be greater than 1 or smaller than 0 , and we shall make use of this liberty if it helps us to escape contradictions. The range of the variables expressed by small letters " $p$," " $q$," and so on, extends therefore through all real numbers, the necessary restrictions for non-empty classes $O$ being expressed by the Axiom II, 2.

As far as I see we need not introduce any further qualifications within the system of probability implication. I should like however to add some remarks concerning the use of the functor $W($ ). A functor is usually conceived as being unambiguous; thus $W(O, P)$ would mean "the probability from $O$ to $P$." Our symbol has this meaning only in case $O$ is not empty. To include the case of $O$ being empty we have to translate every formula of the functor-writing into an existence statement such as explained on pages $61-62$, or in the examples on pages $69-70$ and page 73 . The formula then means, in accordance 
with the rule of existence: if $n-1$ of the $n$ probabilities of the formula exist and have among their values the values $p, q, \cdots$, then also the $n$th probability exists and has among its values a value $w$ such that the $p, q, \cdots, w$ satisfy the equation expressed in the formula. It is obvious that for non-empty classes $O$ this interpretation is identical with the meaning of unambiguous functors. For the case of empty classes $O$ however it also will lead to consistent interpretations although of course in this case the formulae actually state nothing, as all numerical values of the probabilities then are possible. It follows that we need not add the condition $\overline{(\bar{O})}$ to any of the formulae of the functor-writing.

For all practical purposes it is convenient to interpret the functor formulae as referring to a non-empty class $O$, and then to interpret the functor in the usual way as meaning "the probability from ... to ...." Here the term $O$ is defined as that term which occurs in the first place of every probability expression of a formula. It is interesting that this interpretation can also be carried through if some of the other classes are empty, even if they appear in the first place of the probability functor; it turns out that each formula can be written in a form in which the indeterminate probability values are multiplied by 0 such as in (3), p. 73, if we assume $P$ to be empty.

I am much indebted to Mr. J. C. C. McKinsey and to Mr. S. C. Kleene for having pointed out the necessity of the correction of my axioms of probability implication with respect to empty classes $O$.

University of California at Los Angeles 